Sirada Panupattanapong, MD Center for Pediatric Rheumatology and Immunology, Cleveland Clinic Children's
Elizabeth B. Brooks, MD, PhD

Center for Pediatric Rheumatology and Immunology,

Cleveland Clinic Children's

\title{
New spectrum of COVID-19 manifestations in children: Kawasaki-like syndrome and hyperinflammatory response
}

\section{Posted December 29, 2020}

\section{ABSTRACT}

In April 2020, data began emerging on Kawasaki-like syndrome and hyperinflammatory response in children with COVID-19. Since then, much has been published on the presentation of COVID-19 in pediatric patients, including its association with Kawasaki disease and the multisystem inflammatory syndrome in children (MIS-C). However, questions still remain regarding the risk factors, pathogenesis, prognosis, and specific therapy for these manifestations of COVID-19.

\section{INTRODUCTION}

In late April 2020, the United Kingdom's National Health Service issued an alert that heightened awareness of a new COVID-19 manifestation in children - a cluster of previously healthy children with COVID-19 had become ill with overlapping symptoms of Kawasaki disease and toxic shock syndrome with laboratory results showing severe systemic inflammation.

Since then, more cases have been reported, mostly from other European countries and the United States. On May 14, 2020, the New York State Department of Health reported more than 100 cases of children who were hospitalized with this phenomenon. On December 1, that number had increased to 278 with 2 deaths. ${ }^{1}$ Before that, COVID-19 was thought to rarely cause harm to children, with most being asymptomatic or experiencing only mild respiratory symptoms. This showed a need to better understand the impact of COVID-19 on children, especially when it causes critical illness.

The statements and opinions expressed in COVID-19 Curbside Consults are based on experience and the available literature as of the date posted. While we try to regularly update this content, any offered recommendations cannot be substituted for the clinical judgment of clinicians caring for individual patients.

doi:10.3949/ccjm.87a.ccc039

\section{WHAT IS TRUE KAWASAKI DISEASE?}

Kawasaki disease (KD) was first characterized in 1967 by Tomisaku Kawasaki who described a series of Japanese children suffering from "acute febrile mucocutaneous syndrome with lymphoid involvement with specific desquamation of the fingers and toes." ${ }^{2}$ This disease is now known to occur worldwide with higher incidences in Eastern versus Western nations.

Kawasaki disease is an acute, self-limited febrile illness that predominately affects young children, especially those under 5 years of age, with a median age of onset of 9 to 11 months. Approximately 25\% of cases occur in older children, and it rarely affects adults. It is more common in males than females by a ratio of 1.5 to $1 .^{3}$

Despite more than 50 years of study, the exact etiology of KD remains unknown. The current concept is that this syndrome results from an immunologic response to an exposure in the respiratory system or GI tract or both in genetically susceptible children. The immunologic cascade leads to systemic inflammation in medium-sized arteries and multiple organs during the acute phase. Because the incidence of $\mathrm{KD}$ peaks during the winter and spring, it points toward infectious agents as a primary trigger.

\section{Diagnosis}

The symptoms of KD (those not associated with COVID-19) can resemble acute infections such as viral infection, bacterial lymphadenitis, or scarlet fever. The diagnostic criteria for KD are as follows: ${ }^{4}$

- Fever for at least 5 days, and

- The presence of at least 4 of 5 principle clinical features:

1. Extremity changes with erythema and edema of palms and soles during the acute phase. 
Skin peeling can be seen in the convalescent phase.

2. Diffuse polymorphic rash including maculopapular, diffuse erythroderma or erythema multiforme-like.

3. Bilateral nonexudative bulbar conjunctivitis typically sparing the limbus.

4. Oral mucosal changes with cracked lips, oral and pharyngeal erythema, "strawberry tongue" (erythema of tongue with prominent fungiform papillae).

5. Cervical lymphadenopathy, typically unilateral, at least $1.5 \mathrm{~cm}$ in diameter.

Some patients, particularly infants and older children, may have an atypical presentation that does not fulfill the diagnostic criteria (ie, incomplete KD). They may have spontaneous defervescence before 5 days, or prolonged unexplained fever along with only 2 or 3 principle clinical findings. In this scenario, a KD diagnosis can be made if there are at least 3 compatible laboratory findings - anemia, thrombocytosis, hypoalbuminemia, elevated alanine aminotransferase, leukocytosis, or leucouria - or an echocardiogram showing coronary artery dilatation or aneurysm. ${ }^{4}$

\section{Patient management}

Kawasaki disease is the most common cause of acquired heart disease of children in the US and developed countries. Children with KD should receive treatment as $25 \%$ of untreated children can develop coronary artery aneurysm, posing a significant risk of thrombosis and myocardial infarction in later life. ${ }^{4}$ Large aneurysms can rupture and cause death. Severe cases of KD may present with shock state (Kawasaki shock disease syndrome), similar to what has been described currently in association with COVID-19. Rarely, macrophage activation syndrome (MAS)/hemophagocytic lymphohistiocytosis (HLH) can complicate the course.

For classic KD, intravenous immunoglobulin (IVIG) therapy is the cornerstone of treatment along with other adjunct treatments for patients with disease not responsive to IVIG, including corticosteroids, cyclosporine, and biologics (anti-tumor necrosis factor and anti-interleukin [IL]-1). The primary purpose of treatment is to halt the acute systemic inflammatory process to prevent the development of coronary artery changes. Aspirin is given for antithrombotic effect.

\section{WHAT WE KNOW ABOUT COVID-19-ASSOCI- ATED KD-LIKE SYNDROME AND OTHER MANI- FESTATIONS}

It is unclear if COVID-19 causes KD or a syndrome that mimics this condition. Most data were generated from an online meeting of European and US investigators in early May 2020. Groups from both the United Kingdom and Italy have published case series describing children with this multisystem inflammatory syndrome. ${ }^{5,6}$

In the UK series, ${ }^{5} 8$ previously healthy children presented with vasoplegic shock and myocardial dysfunction, 5 males and 6 of Afro-Caribbean descent. All had unrelenting fever along with some features seen in patients with KD (rash, conjunctivitis, peripheral edema). Interestingly, all had prominent GI symptoms including nonbloody diarrhea, abdominal pain, and vomiting, and imaging showed ascites and ileitis. One child developed giant coronary aneurysm, and one died from refractory shock and a large cerebrovascular infarction.

The Italian researchers ${ }^{6}$ presented data on 10 children from the Bergamo province in Italy, which had a high rate of SARS-CoV-2 infections at that time. They reported a 30-fold increased monthly incidence of KD from February 18, 2020 to April 20, 2020 compared with the previous 5 years. Of the 10 children, 7 were male, 5 presented with classic KD, 5 were classified as incomplete $\mathrm{KD}$, and 5 patients met the criteria for Kawasaki shock disease syndrome and MAS.

Laboratory findings in both cohorts showed neutrophilia, lymphopenia, thrombocytopenia, and marked elevation of inflammatory markers. In addition, elevated levels of ferritin, triglycerides, and D-dimer were seen, suggesting MAS/HLH. Most of the patients had significantly elevated levels of proBNP (B-type natriuretic peptide) or troponin- $T$ or both suggesting compromised cardiac function or shock state or both.

Current data show that the emerging phenotypes are a combination of typical or atypical KD, Kawasaki shock syndrome, toxic shock syndrome, and MAS/ HLH. Common presentations are fever, rash, and GI symptoms including abdominal pain, diarrhea, and vomiting. Unlike the adult presentation of COVID19, most children have no significant respiratory involvement. Features of KD, such as mucocutaneous inflammation (eg, conjunctivitis, rash, cervical lymphadenopathy) and systemic inflammation involving single or multiple organs (eg, liver, renal, neurological), can be seen. A subset of patients develop coronary artery aneurysms, as seen with $\mathrm{KD}$. 
Severe cases presented with shock as a result of cardiac dysfunction with or without myocarditis in the setting of a hyperinflammatory state and required inotropic treatment with features resembling Kawasaki shock and toxic shock syndrome. This phenomenon appears to result from an uninhibited immune response to a prior SARS-CoV-2 infection rather than a direct injury resulting from an acute viral infection. This is speculated from the surge of cases that occurred about 2 to 3 weeks after the peak of infection in their area and that the majority of these children had negative COVID-19 results on a polymerase chain reaction nasopharyngeal swab test but had positive viral serology results. They were previously fit and well, without preceding COVID-19 symptoms, but some had a history of contact with a COVID-19 patient.

In comparison to classic $\mathrm{KD}$, the predilection for male gender is similar; however, this syndrome appears to affect older children (aged 5 to 14 years) rather than the younger age group. These patients also have lower white blood cell counts, lower lymphocyte and platelet counts, higher ferritin levels, and elevated cardiac markers. ${ }^{6}$ In general, the incidence of classic $\mathrm{KD}$ is higher in children of Asian descent; however, in the UK cohort, more than $50 \%$ of those with the KD-like syndrome were of Afro-Caribbean descent. ${ }^{5}$ This observation may suggest a genetic susceptibility in this subgroup or reflect a higher rate of COVID-19 in this population.

The provisional name coined for this KD-like syndrome by UK experts is "pediatric multisystem inflammatory syndrome temporally associated with SARS-CoV-2 (PMIS-TS)."7 The Centers for Disease Control and Prevention named this condition "multisystem inflammatory syndrome in children (MISC)." ${ }^{8}$ It is defined in Table 1.

\section{MANAGEMENT OF MIS-C ASSOCIATED WITH COVID-19}

This is an evolving spectrum of COVID-19 in the pediatric population, and there is much to learn. It is important to reiterate that MIS-C is rare and has only occurred in a small number of children. Early recognition is crucial as these patients may be critically ill and require care in a pediatric intensive care unit as well as evaluation from multiple specialties including infectious disease, cardiology, hematology/oncology, and rheumatology.

At present [original article published May 28, 2020], there is no standardized treatment guideline for MIS-C.
TABLE 1

\section{Case definition for Multisystem Inflammatory Syndrome in Children (MIS-C)}

An individual $<21$ years presenting with

- $\quad$ Fever, ${ }^{a}$ laboratory evidence of inflammation, ${ }^{b}$ and evidence of clinically severe illness requiring hospitalization, with multisystem ( $>2$ ) organ involvement (cardiac, renal, respiratory, hematologic, gastrointestinal, dermatologic or neurological); AND

- $\quad$ No alternative plausible diagnoses; AND

- Positive for current or recent SARS-CoV-2 infection by RT-PCR, serology, or antigen test; or COVID-19 exposure within the 4 weeks prior to the onset of symptoms

Additional comments

- Some individuals may fulfill full or partial criteria for Kawasaki disease but should be reported if they meet the case definition for MIS-C

- $\quad$ Consider MIS-C in any pediatric death with evidence of SARS-CoV-2 infection

\footnotetext{
${ }^{\text {a }}$ Fever $>38.0^{\circ} \mathrm{C}$ for $\geq 24$ hours, or report of subjective fever lasting $\geq 24$ hours.

bIncluding, but not limited to, 1 or more of the following: an elevated C-reactive protein, erythrocyte sedimentation rate, fibrinogen, procalcitonin, d-dimer, ferritin , lactic acid dehydrogenase, or interleukin 6 , elevated neutrophils, reduced lymphocytes and low albumin.

RT-PCR = reverse transcription polymerase chain reaction Reproduced from Centers for Disease Control and Prevention website.?
}

Treatment strategies depended on where the patient fits in the disease spectrum and the clinical experiences of the treating practitioners.

Based on case reports, patients meeting KD or atypical KD criteria or those with coronary artery changes should receive IVIG at $2 \mathrm{~g} / \mathrm{kg}$. High-dose aspirin has been used in conjunction with IVIG ${ }^{5,6}$ If hyperinflammatory features are prominent, immunosuppression can be considered, including corticosteroids or biologic medications such as anakinra (anti-IL-1) or tocilizumab (anti-IL-6). Upregulation of the IL-1 signaling pathway is a highlighted mechanism in both MAS and KD, supporting the use of anakinra in this setting. ${ }^{9,10}$

Anticoagulation can be considered if D-dimer levels are markedly elevated, given the hypercoagulability and clot formation seen in adults with COVID-19. ${ }^{11,12}$ Cardiogenic shock and myocardial compromise can lead to fatal outcomes. Vigilant cardiac monitoring, checking serial cardiac markers (pro-BNP, troponin), and reviewing echocardiograms (focusing on cardiac function and coronary arteries) are also crucial. 


\section{ADDENDUM}

Since the original publication of this article in May 2020, much more has been learned about the presentation of COVID-19 in pediatric patients, including its association and differences between MIS-C and $\mathrm{KD}$, summarized in Table 2 . There have been numerous publications from around the world on this topic, and we update some of the information below.

\section{CARDIAC COMPLICATIONS SEEN WITH COVID-19 AND MIS-C}

A study from the Children's Hospital of Philadelphia reviewed data on echocardiograms done in 28 pediatric patients with a clinical diagnosis of MIS-C from April 10 to June 7, 2020. ${ }^{17}$ Researchers noted that coronary artery dilatation and ectasia were rare in the patients; however, myocardial dysfunction was more common with both left ventricular systolic and diastolic dysfunction. When patients with classic KD without associated COVID-19 were compared with patients with MIS-C, there was worse cardiac function in the MIS-C group. Because the study population is small, conclusions cannot be made regarding the low number of patients with coronary artery dilatation or coronary ectasia.

A study from Cohen Children's Medical Center in Queens, New York, found a greater number of patients with coronary artery abnormalities. ${ }^{18}$ In their cohort of 33 patients admitted from April 17 to May 13, 2020, 48\% (16 patients) had coronary artery abnormalities on ECG and LV dysfunction in 58\% (19). All patients tested positive for SARS-CoV-2.

A single-center observational study from the United Kingdom ${ }^{19}$ reported that $9(60 \%)$ of 15 children with PMIS-TS had abnormal findings on electrocardiogram, which normalized in 6 of them before hospital discharge. Overall, 93\% (14 patients) had coronary artery abnormalities: 7 with prominent coronary arteries on echocardiogram but with coronary measurements within normal; 6 with coronary ectasia with increased $z$ scores in left main or left anterior descending arteries; 1 with fusiform aneurysms involving the right coronary and left anterior descending arteries. Of note, $7 \%$ of the children developed their abnormalities on day 5 of hospitalization, which had been normal on admission. In $43 \%$ (6), the coronaries normalized at a median of 3 days, but the remaining continued to have abnormalities at discharge. Left ventricular dysfunction was also reported in $80 \%$ of their patient cohort with $75 \%$ of the abnormalities being seen at presentation. ${ }^{19}$
Feldstein et $\mathrm{al}^{20}$ conducted a multicenter collaboration study of 186 pediatric patients diagnosed with MIS-C in 26 different US pediatric centers. States with the most cases were New York, New Jersey, and Michigan. In this cohort, 70\% (131) of the patients tested positive for SARS-CoV-2. There was cardiac involvement in 80\% (149), and 8\% (15) had coronary-artery aneurysms. Most patients (73\%) had elevated brain natriuretic peptide (BNP) levels, and $50 \%$ had elevated troponin levels. Vasopressor support was required in $48 \%$ (90) of the patients. Nearly all patients $(91 \%)$ had at least one echocardiogram. Presence of pericardial effusion or pericarditis was found in $26 \%$. Left ventricular dysfunction was reported in 38\% based on an ejection fraction of less than $55 \%$.

A review of data from the New York State Department of Health on 99 hospitalized pediatric patients with MIS-C found that $52 \%$ had cardiac dysfunction, $32 \%$ had pericardial effusion, and $9 \%$ had coronary artery aneurysms. ${ }^{21}$ Kawasaki disease was diagnosed in 36 patients. These patients with $\mathrm{KD}$ were overall younger, with $91.7 \%$ aged from 0 to 12 years and the remainder aged 13 to 20 years. In contrast, myocarditis was seen most often in children aged 6 to 20 years with the highest number of cases in those aged 6 to 12 years. $^{21}$

In a case report, an 11-year-old child with MIS-C who presented with cardiogenic shock and died within 28 hours of admission was found to have myocarditis, pericarditis, and endocarditis on autopsy with widespread inflammation and necrosis of cardiac myocytes. ${ }^{22}$ SARS-CoV-2 was identified in the myocardial tissue in cardiac myocytes as well as in endothelial cells in the endocardium. Viral particles also were found in other inflammatory cells and mesenchymal cells in the heart. The authors suggest that there is a primary infection of cardiac myocytes that leads to an inflammatory reaction, and that the presence of the infection and inflammatory reaction together leads to necrosis of cardiac myocytes. ${ }^{22}$

There has been much debate regarding $\mathrm{KD}$ and cardiac findings in MIS-C and whether they are the same or separate entities, as the cause of KD has yet to be identified but is believed to be triggered by a preceding infection. There is much to learn from patients with SARS-CoV-2 and inflammatory sequelae.

\section{GASTROINTESTINAL INVOLVEMENT SEEN WITH MIS-C AND COVID-19}

Gastrointestinal symptoms are prominent in patients with MIS-C and can cause confusion with patients 
TABLE 2

\section{Comparison of MIS-C and Kawasaki disease in children}

\begin{tabular}{|c|c|c|}
\hline & MIS-C & Kawasaki disease \\
\hline Median age, year (IQR) & $9(5.7-14)$ & $2.7(1.4-4.7)$ \\
\hline High-risk population & $\begin{array}{c}\text { African American, } \\
\text { Hispanic, Afro- } \\
\text { Caribbean }\end{array}$ & Asian \\
\hline Gastrointestinal symptoms & +++ & + \\
\hline Shock & ++ & + \\
\hline Low ejection fraction & $\downarrow \downarrow$ & Normal or $\downarrow$ \\
\hline Coronary abnormality & + & ++ \\
\hline Intensive care support & + & ++ \\
\hline White blood cell & $\uparrow \uparrow$ & $\uparrow$ \\
\hline Neutrophil & $\uparrow \uparrow$ & $\uparrow$ \\
\hline Lymphocyte & $\downarrow \downarrow$ & Normal \\
\hline Platelet & $\downarrow$ & $\uparrow \uparrow$ \\
\hline CRP & $\uparrow \uparrow \uparrow$ & $\uparrow$ \\
\hline Ferritin & $\uparrow \uparrow$ & Normal or $\uparrow$ \\
\hline NT-proBNP & $\uparrow \uparrow \uparrow$ & Normal or $\uparrow$ \\
\hline Troponin & $\uparrow$ & Normal or $\uparrow$ \\
\hline D-dimer & $\uparrow \uparrow \uparrow$ & $\uparrow$ \\
\hline
\end{tabular}

$\mathrm{CRP}=\mathrm{C}$-reactive protein; $\mathrm{IQR}=$ interquartile range; $\mathrm{MIS}-\mathrm{C}=$ multisystem inflammatory syndrome in children; NT-proBNP $=\mathrm{N}$-terminal-proB-type natriuretic peptide

Adapted from references 13-16.

noted in $91 \%$ to $93 \%$ of all patient age groups $(<$ 5 years to 20 years). ${ }^{20}$

\section{TREATMENT}

There has been more research published to guide the management of MIS-C in pediatric patients. The American College of Rheumatology (ACR) published a Task Force recommendation for patients with MIS-C in June $2020^{24}$ and updated it in December 2020.25 There are no comparative studies among therapies to treat MIS-C; therefore, the clinical guidance is based on current evidence and expert opinions. Patients with mild symptoms of MIS-C account for $22 \%$ of the cases, ${ }^{13}$ and the ACR recommendations suggest that they may not need an immunomodulator and require only close monitoring. ${ }^{25}$

Stepwise progression of immunomodulatory therapy should be used based on clinical severity. At present, the Task Force recommends IVIG (1-2 $\mathrm{mg} / \mathrm{kg} /$ day, based on ideal body weight) be given to ALL MIS-C patients who require hospitalization. Glucocorticoids should be used as adjunctive therapy with IVIG in patients who present with ill appearance, marked elevated of BNP, unexplained tachycardia, shock, or organthreatening disease (1-2 mg/kg/day). Children who require inotropes/and or vasopressors may require higher doses $(10-30 \mathrm{mg} / \mathrm{kg} /$ day $)$. In refractory cases, anakinra (recombinant human

presenting with acute COVID-19 infection.

- In a cohort of 44 pediatric patients from the Children's Hospital at Columbia University, New York, New York, 84\% presented with GI symptoms, predominately abdominal pain $(75 \%)$, vomiting (57\%), and diarrhea (49\%). ${ }^{23}$ All patients had documented SARS-CoV-2 infection. Elevated transaminases were reported in $52 \%$ of patients. Elevated transaminases also were reported in adult patients presenting with an inflammatory syndrome associated with SARS-CoV-2 infection but to a lesser extent. ${ }^{23}$

- In the New York state-wide cohort, ${ }^{21}$ similar percentages were reported for GI symptoms: $80 \%$ with any GI symptom, $60 \%$ with abdominal pain, $58 \%$ with nausea or vomiting, and $49 \%$ with diarrhea. When evaluated by age, the percentages were similar across the different age groups, in contrast to cardiac disease which was higher in the older age groups.

- In the multicenter US cohort, GI symptoms were
IL-1 receptor antagonist) doses of more than $4 \mathrm{mg} /$ $\mathrm{kg} /$ day can be considered. The second dose of IVIG is not recommended given the risk of IVIG complications such as hemolytic anemia and volume overload. Once clinical improvement is seen, immunomodulators should be tapered over 2 to 3 weeks (or longer) to avoid rebound flare of inflammation. ${ }^{24,25}$

To reduce the risk of thrombosis in patients with MIS-C and KD-like features and/or thrombocytosis, use low-dose aspirin (3-5 mg/kg/day; max $81 \mathrm{mg} /$ day) and continue it until the platelet count has normalized or there is confirmation of normal coronary arteries at least 4 weeks after diagnosis. If giant coronary aneurysms (coronary z score $\geq 10$ ) are seen, therapeutic anticoagulation with enoxaparin or warfarin to prevent thromboembolic events is recommended. ${ }^{24,25}$

A survey of inpatient management of MIS-C was conducted by the International Kawasaki Disease Registry with respondents from 38 institutions in 11 countries. ${ }^{26}$ Approximately half reported using immunomodulatory treatment for all patients with MIS-C 
regardless of presentation, with the others treating only severe cases. Of respondents, 53\% and 14\% used IVIG or steroids, respectively, in all patients regardless of clinical presentation. The rest reported selective use of IVIG in specific circumstance including presence of KD clinical features, coronary artery involvement, myocardial involvement, and severe clinical presentation such as intensive care unit admission or shock.

Steroids were used most frequently in patients with severe clinical presentations or in those who did not respond to IVIG. Other indications for steroid use included the presence of valve dysfunction, MAS, and high risks of developing coronary artery aneurysm. Biologic therapies were used as an adjunct in severe or refractory case, including anakinra (58\%), infliximab (28\%), and tocilizumab (8\%). Overall, 91\% used anti-inflammatory dosing of acetylsalicylic acid (aspirin) (30-50 or $80-100 \mathrm{mg} / \mathrm{kg} /$ day), stating it is indicated in patients with $\mathrm{KD}$ features and/or coronary artery ectasia or aneurysm. Overall, 17\% did not use anti-inflammatory medications. ${ }^{26}$

A group from France has reported a potential benefit on cardiac outcome from a combination of IVIG and IV methylprednisolone compared with IVIG alone in patients with MIS-C. ${ }^{27}$ The 18 patients admitted before May 12020 received IVIG $2 \mathrm{~g} / \mathrm{kg}$ alone and 22 patients admitted after this date received a combination of IVIG and IV methylprednisolone therapy $(0.8 \mathrm{mg} / \mathrm{kg} /$ day during 5 days $)$. The combination therapy group had significantly reduced time to recovery of left ventricular ejection fraction ( 2.9 vs 5.4 days), isovolumic relaxation time ( 6.4 vs 20.6 days), and pediatric intensive care unit stay (3.4 vs 5.4 days). However, a major limitation of this study is the absence of randomization. ${ }^{27}$

\section{Conclusion}

Treatment for MIS-C is individualized and varies among healthcare practice and institutions. Therapeutic approaches have been extrapolated from treatment of KD, toxic shock syndrome, and cytokine storm in patients with COVID-19. Currently, there is no optimal study comparing these different treatment approaches for MIS-C patients.

\section{DISTINCT CLINICAL FEATURES AND IMMUNOLOGIC FEATURES OF MIS-C VS KAWASAKI DISEASE}

There is growing evidence that MIS-C is distinct from KD although they have some clinical overlap and both respond well to IVIG treatment. There could be differences in genetic composition as MIS-C has higher prevalence in African American, Hispanic, and Afro-Caribbean populations while $\mathrm{KD}$ is more common in Asian populations. Also, MIS-C presents more in older children and has more GI manifestations, lymphopenia, and thrombocytopenia along with elevated levels of C-reactive protein, pro-BNP, ferritin, and D-dimer. ${ }^{14}$

However, not all MIS-C patients have KD clinical features. In a study from the Boston Children's Hospital, Massachusetts, only $25 \%$ of 28 patients with MIS-C met the criteria for either complete or incomplete KD. ${ }^{15}$ The prevalence of coronary artery abnormalities was significantly lower in MIS-C patients $(21 \%)$ than in the KD group (35\%), but the MIS-C patients had significantly lower ejection fraction. Also, MIS-C was distinct from MAS as MIS-C patients had less striking increase in soluble IL-2 receptor, ferritin, IL-18, and CXCL9 (a surrogate marker for interferon gamma) than those with MAS.

\section{DISCLOSURES}

The authors report no relevant financial relationships which, in the context of their contributions, could be perceived as a potential conflict of interest.

\section{REFERENCES}

1. New York State Department of Health. Childhood inflammatory disease related to COVID-19. https://coronavirus.health.ny.gov/ childhood-inflammatory-disease-related-covid-19. Accessed on December 23, 2020.

2. Kawasaki T. Acute febrile mucocutaneous syndrome with lymphoid involvement with specific desquamation of the fingers and toes in children. Article in Japanese. Arerugi 1967; 16(3):178-222. pmid: 6062087

3. Holman RC, Belay ED, Christensen KY, Folkema AM, Steiner CA, Schonberger LB. Hospitalizations for Kawasaki syndrome among children in the United States, 1997-2007. Pediatr Infect Dis J 2010; 29(6):483-488. doi:10.1097/INF.0b013e3181cf8705

4. McCrindle BW, Rowley AH, Newburger JW, et al. Diagnosis, treatment, and long-term management of Kawasaki disease: a scientific statement for health professionals from the American Heart Association. [Published correction appears in Circulation 2019; 140(5):e181-e184]. Circulation 2017; 135(17):e927-e999. doi:10.1161/CIR.0000000000000484

5. Riphagen S, Gomez X, Gonzalez-Martinez C, Wilkinson N, Theocharis P. Hyperinflammatory shock in children during COVID-19 pandemic. Lancet 2020; 395(10237):1607-1608. doi:10.1016/ S0140-6736(20)31094-1

6. Verdoni L, Mazza A, Gervasoni A, et al. An outbreak of severe Kawasaki-like disease at the Italian epicentre of the SARS-CoV-2 epidemic: an observational cohort study. Lancet 2020; 395(10239):1771-1778. doi:10.1016/S0140-6736(20)31103-X

7. Royal College of Paediatrics and Child Health. Guidance: Paediatric multisystem inflammatory syndrome temporally associated with COVID-19. May 1, 2020. https://www.rcpch.ac.uk/sites/default/ files/2020-05/COVID-19-Paediatric-multisystem-\%20infl ammatory\%20syndrome-20200501.pdf. Accessed May 27, 2020.

8. Centers for Disease Control and Prevention. Multisystem inflammatory syndrome in children (MIS-C) associated with coronavirus 
disease 2019 (COVID-19). CDC Health Alert Network publication CDCHAN-00432. May 14, 2020. https://emergency.cdc.gov/han/2020/ han00432.asp. Accessed May 27, 2020.

9. Sönmez HE, Demir S, Bilginer Y, Özen S. Anakinra treatment in macrophage activation syndrome: a single center experience and systemic review of literature. Clin Rheumatol 2018; 37(12):3329-3335. doi:10.1007/s10067-018-4095-1

10. Tremoulet $\mathrm{AH}$. Adjunctive therapies in Kawasaki disease. Int J Rheum Dis 2018; 21(1):76-79. doi:10.1111/1756-185X.13208

11. Klok FA, Kruip MJHA, van der Meer NJM, et al. Incidence of thrombotic complications in critically ill ICU patients with COVID-19. Thromb Res 2020; 191:145-147. doi:10.1016/j.thromres.2020.04.013 [updated reference] Klok FA, Kruip MJHA, van der Meer NJM, et al. Confirmation of the high cumulative incidence of thrombotic complications in critically ill ICU patients with COVID-19: an updated analysis. Thromb Res 2020; 191:148-150. doi: 10.1016/j. thromres.2020.04.041.

12. Poissy J, Goutay J, Caplan M, et al. Pulmonary embolism in COVID-19 patients: awareness of an increased prevalence. Circulation 2020; 142(2)L184-186. doi:10.1161/CIRCULATIONAHA.120.047430

13. Whittaker E, Bamford A, Kenny J, et al. Clinical characteristics of 58 children with a pediatric inflammatory multisystem syndrome temporally associated with SARS-CoV-2. JAMA 2020; 324(3):259-269. doi:10.100/jama.2020.10369

14. Loke $\mathrm{YH}$, Berul $\mathrm{Cl}$, Harahsheh AS. Multisystem inflammatory syndrome in children: is there a linkage to Kawasaki disease? Trends Cardiovasc Med 2020; 30(7):389-396. doi:10.1016/j.tcm.2020.07.004

15. Lee PY, Day-Lewis $M$, Henderson LA, et al. Distinct clinical and Immunological Features of SAR-COV2 induced multisystem inflammatory syndrome in children. J Clin Invest 2020; 130(11):5942-5950. doi:10.1172/JCl141113

16. Toubiana J, Poirault C, Corsia A, et al. Kawasaki-like multisystem inflammatory syndrome in children during the covid-19 pandemic in Paris, France: prospective observational study. BMJ 2020; 369:m2094. doi:10.1136/bmj.m2094.

17. Matsubara D, Kauffman HL, Wang $Y$, et al. Echocardiographic findings in pediatric multisystem inflammatory syndrome associated with COVID-19 in the United States. J Am Coll Cardiol 2020; 76(17):1947-1961. doi:10.1016/j.acc.2020.08.056

18. Capone CA, Subramony A, Sweberg T, et al. Characteristics, cardiac involvement, and outcomes of multisystem inflammatory syndrome of childhood associated with severe acute respiratory syndrome coronavirus 2 infection. J Pediatr 2020; 224:141-145. doi:10.1016/j. peds.2020.06.044

19. Ramcharan T, Nolan $O$, Lai $C Y$, et al. Paediatric inflammatory multisystem syndrome: temporally associated with SARS-CoV-2 (PIMS-TS): cardiac features, management and short-term outcomes at a UK tertiary paediatric hospital. Pediatr Cardiol 2020; 41(7):1391-1401. doi:10.1007/s00246-020-02391-2

20. Feldstein LR, Rose EB, Horwitz SM, et al. Multisystem inflammatory syndrome in U.S. children and adolescents. N Engl J Med 2020; 383(4):334-346. doi:10.1056/NEJMoa2021680

21. Dufort EM, Koumans EH, Chow EJ, et al. Multisystem inflammatory syndrome in children in New York State. N Engl J Med 2020: 383(4):347-358. doi:10.1056/NEJMoa2021756

22. Dolhnikoff M, Ferreira Ferranti J, de Almeida Monteiro RA, et al. SARS-CoV-2 in cardiac tissue of a child with COVID-19-related multisystem inflammatory syndrome. Lancet Child Adolesc Health 2020; 4(10):790-794. doi:10.1016/\$2352-4642(20)30257-1

23. Miller J, Cantor A, Zachariah P, et al. Gastrointestinal symptoms as a major presentation component of a novel multisystem inflammatory syndrome in children that is related to coronavirus disease 2019: a single center experience of 44 cases. Gastroenterology 2020; 159(4):1571-1574.e2. doi:10.1053/j.gastro.2020.05.079

24. Henderson LA, Canna SW, Friedman KG, et al. American College of Rheumatology clinical guidance for multisystem inflammatory syndrome in children associated with SARS-CoV-2 and hyperinflammation in pediatric COVID19: Version 1. Arthritis Rheumatol 2020; 72(11):1791-1805. doi:10.1002/art.41454
25. Henderson LA, Canna SW, Friedman KG, et al. American College of Rheumatology Clinical guidance for pediatric patients with multisystem inflammatory syndrome in children (MIS-C) associated with SARS-CoV-2 and hyperinflammation in COVID-19. Version 2. Arthritis Rheumatol 2020 Dec 5. doi:10.1002/art.41616

26. Elias M, McCrindle BW, Larios $G$, et al. Management of multisystem inflammatory syndrome in children associate with COVID-19: a survey from the International Kawasaki Disease Registry. CJC Open 2020; 2(6):632-640. doi:10/1016/j.cjco.2020.09.004

27. Belhadjer Z, Auriau J, Meot M, et al. Addition of corticosteroids to immune globulins is associated with recovery of cardiac function in multi-inflammatory syndrome in children (MIS-C). Research letter. Circulation 2020; 142(23):2282-2284. doi:10.1161/circulation. aha.120.050147

Address: Sirada Panupattanapong, MD, Center for Pediatric Rheumatology and Immunology, R3, Cleveland Clinic, 9500 Euclid Avenue, Cleveland, $\mathrm{OH} 44195 ;$ panupas@ccf.org 